\title{
A Study of the Reliability and Validity of the Korean Version of DSM-5 Symptom Measure-Inattention and Anger for Parent and Guardian of Child Age 6 to 17
}

\author{
Min-Sup Shin ${ }^{1 *}$, Bung-Nyun Kim*, Minji Cho², Mirae Jang ${ }^{2}$, Hanbyul Shin ${ }^{2}$, \\ Ryemi Do ${ }^{3}$, Hyungseo Park ${ }^{4}$, Narae Yoon ${ }^{4}$, Gahye Noh ${ }^{4}$, Jae-Won Song ${ }^{4}$, \\ Yebin $\mathrm{Ahn}^{4}$, Jiyoon $\mathrm{Shin}^{4}$, Soomin Jang ${ }^{4}$, Eunjung $\mathrm{Noh}^{2}$, and Eunhwa Lee ${ }^{2}$ \\ ${ }^{1}$ Department of Psychiatry, College of Medicine, Seoul National University, Seoul, Korea \\ ${ }^{2}$ Biomedical Research Institute, Seoul National University, Seoul, Korea \\ ${ }^{3}$ Department of Clinical Medical Sciences, College of Medicine, Seoul National University, Seoul, Korea \\ ${ }^{4}$ Department of Child and Adolescent Psychiatry, Seoul National University Hospital, Seoul, Korea
}

\begin{abstract}
Objectives: This study was conducted to investigate the reliability and validity of the Korean version of the DSM-5 Level 2 Cross-Cutting Symptom Measure-inattention [Swanson, Nolan and Pelham, version IV (SNAP-IV)] and anger [Patient-Reported Outcome Measurement Information System (PROMIS) Anger] for parents and guardians of children aged 6-17 years.

Methods: We included 104 children and adolescents diagnosed with attention-deficit/hyperactivity disorder (ADHD), ADHD with anxiety and depression, depressive disorder, anxiety disorder, and tic disorder with somatic symptoms (ADHD=41, depression=9, anxiety=14, ADHD+anxious depression=11, tic+somatic symptoms=29). Their ages ranged from 8 years to 15 years. The participants' mothers completed the SNAP-IV, PROMIS Anger scale, Korean version of the IOWA Conners Rating Scale (K-IOWA), and Korean ADHD Rating Scale (K-ARS) so that the reliability and validity of the SNAP-IV and PROMIS Anger scales, which are DSM-5 scales for assessing inattention and anger of children and adolescents, could be examined.

Results: The reliability coefficient of SNAP-IV (Cronbach's $\alpha$ ) was 0.94 . The correlation coefficients between SNAP-IV, K-IOWA inattention, and K-ARS inattention scores ranged from 0.73 to 0.86. The mean SNAP-IV scores of the ADHD and the ADHD+anxious depression groups were significantly higher than those of the anxiety and the tic+somatic symptoms groups. The reliability coefficient of the PROMIS Anger was 0.91. The correlation coefficient between PROMIS Anger and K-IOWA oppositional/defiant scores was 0.75. The PROMIS Anger mean score of the ADHD+anxious depression group tended to be higher than that of the other groups.

Conclusion: These results suggest that the Korean version of the DSM-5 Level 2 Cross-Cutting Symptom Measure-inattention and anger for parent and guardian of child age 6-17 might be a reliable and valid test and may be useful for screening children and adolescents with ADHD.
\end{abstract}

Key Words: Attention deficit disorder with hyperactivity; Inattention; Anger; Patient reported outcome measures; Reliability; Validity.

Received: November 27, 2020 / Revision: January 14, 2021 / Accepted: January 27, 2021

Address for correspondence: Min-Sup Shin, Department of Psychiatry, Seoul National University College of Medicine, 101 Daehak-ro, Jongno-gu, Seoul 03080, Korea

Tel: +82-2-2072-2454, Fax: +82-2-744-7241, E-mail: shinms@snu.ac.kr

*These authors contributed equally to this work.

\section{INTRODUCTION}

A considerable number of children and adolescents visiting the department of child and adolescent psychiatry complain of attention problems. According to studies conducted in Korea, $5.9-8.5 \%$ of children and adolescents are known to have attention-deficit/hyperactivity disorder (ADHD) [1], and the number of patients diagnosed with ADHD is rapid-

This is an Open Access article distributed under the terms of the Creative Commons Attribution Non-Commercial License (https://creativecommons.org/licenses/by-nc/4.0) which permits unrestricted non-commercial use, distribution, and reproduction in any medium, provided the original work is properly cited. ly increasing [2]. ADHD is a neurodevelopmental disorder that occurs during childhood and adolescence, accompanied by inattention, impulsivity, and hyperactive behaviors. The main characteristics include problems in selective and sustained attention [3].

Attention in children and adolescents is critical as it is closely associated with academic performance, and many children and adolescents with ADHD tend to show a low level of academic performance. They find it difficult to complete home assignments and prepare for classes at school, and their inattentive and impulsive behaviors prevent them from achiev- 
ing a high level of performance in examinations [4]. In a study on the parents of adolescents with ADHD, one of the most urgent problems for which they sought solutions was academic achievement, which implied that children and adolescents with ADHD as well as their parents regarded the subjective experience of academic problems as a highly serious matter in their daily lives [5]. Moreover, repeated low achievements or academic failure, when combined with hopelessness, low self-esteem, and negative stigma at school, often caused a secondary emotional problem [6]. Among a variety of emotional problems, anger, in particular, is known to be most frequently experienced by children and adolescents with ADHD. While anger is a natural feeling experienced in daily life, uncontrolled anger could cause physical or psychological problems. Numerous studies have reported that compared to normal children and adolescents, those with ADHD showed a high level of physical and verbal aggression with a markedly strong expression of anger $[7,8]$. Notably, as uncontrolled anger could potentially lead to various problems related to violent or defiant behaviors [9], care should be taken regarding secondary emotional problems alongside attention problems exhibited by children and adolescents with ADHD. Therapeutic intervention should be developed based on accurate assessments of ADHD.

However, problematic behaviors, including inattention and anger during childhood and adolescence, are also observed in various other psychiatric disorders such as depression, bipolar disorder, oppositional defiant disorder, and conduct disorder. In addition, the symptoms and therapeutic interventions may vary according to specific disorders or types. Therefore, it is very important to differentially diagnose ADHD and other disorders using accurate assessment tools. Thus, in actual clinical settings, assessments are made objectively and systematically through various methods regarding inattention, impulsivity, and anger control problems in children and adolescents, such as self-reporting questionnaires, rating scales for parents and guardians, clinical interviews, and psychological tests.

Among the tests for screening ADHD, the rating scale for parents and guardians is one of the most widely used tests as it is easy to perform and allows simple scoring and interpretation while reducing the possibility of under-rating that may occur in self-reporting questionnaires based on the ratings of children and adolescents with ADHD themselves due to inadequate self-introspection or evaluation abilities [10]. Moreover, it has been reported that for children and adolescents with ADHD, information from parents or guardians who are able to observe the child or adolescent every day is crucial; it is an essential tool for ADHD diagnosis and treatment together with the self-reporting questionnaires $[11,12]$. The rating scales for parents and guardians most commonly used in Korea today for assessing inattention, anger, and hyperactivity in children and adolescents with ADHD include the ADHD Rating Scale standardized in Korea [13] known as the Korean ADHD Rating Scale (K-ARS) [14], the Korean version of the IOWA Conners Rating Scale (K-IOWA) [15], and the Abbreviated Conners Rating Scale [16].

The DSM-5 Level 2 Cross-Cutting Symptom Measure-inattention [Swanson, Nolan and Pelham, version IV (SNAP-IV)] and anger [Patient-Reported Outcome Measurement Information System (PROMIS) Anger] [1,17]. A study on pediatric subjects in the US reported high reliability for the scales [18], and the assessment tool is frequently used not only in clinical settings but also in clinical research. Shin et al. [19] translated SNAP-IV inattention and PROMIS Anger scales to Korean, and their content validity was verified in a preliminary study. In another study conducted in Korea, these scales were reported to be a reliable tool to measure psychological stress in children and adolescents [20]. In addition, as the SNAP-IV inattention scale and PROMIS Anger scale of the DSM- 5 contain a small number of questions, they are expected to be useful in clinical settings for assessing the symptoms of inattention and anger in children and adolescents in a time-efficient manner and in the follow-up assessment of treatment effects and progress. The use of the scales has thus gradually increased among researchers in Korea [21]. Nevertheless, the reliability and validity of the scales are yet to be verified. Therefore, the present study investigated the reliability and validity of the Korean version of the DSM-5 Level 2 Cross-Cutting Symptom Measure-inattention and anger for parents and guardians of children aged 6-17 years, by targeting children and adolescents of various clinical groups with the aim of developing a reliable and valid standard outcome measure that can be used in ADHD screening and treatment evaluation in Korean clinical settings.

\section{METHODS}

\section{Subjects}

We recruited children and adolescents aged $8-15$ years and their parents who were visiting the department of child and adolescent psychiatry located in Seoul, Korea, during the period from January to December 2020. Based on the diagnosis of child psychiatrists using DSM-5 diagnostic criteria and the comprehensive psychological assessment of a licensed child clinical psychologist, the participants were classified as having $\mathrm{ADHD}, \mathrm{ADHD}+$ anxious depression, depressive disorder, anxiety disorder, and tic disorder+somatic symptoms and were categorized into five groups accordingly. Only participants whose overall Korean Wechsler Intelligence Scale 
for Children, fourth edition scores were $\geq I Q 70$ and $\geq$ Verbal Comprehension Index 80 were recruited in this study, and a total of 104 participants were included in the analysis. This study was conducted after permission was obtained from the Institutional Review Board of Seoul National University Hospital (IRB No. 1950-145-1030).

\section{Assessment tools}

\section{The DSM-5 Level 2 Cross-Cutting Symptom}

Measure-inattention for parents and guardians (SNAP-IV)

SNAP is a scale developed by Swanson based on the ADHD diagnostic criteria of DSM-III [22], while its reconstruction based on the diagnostic criteria of DSM-IV led to SNAP-IV [23]. In this study, the Korean version of the scale, as translated by Shin et al. [19], was used. SNAP-IV is a rating scale for parents and guardians regarding attention-related difficulties observed in children and adolescents aged 6-17 years throughout classes, home assignments, reading, and playing during 2 weeks. It includes eight questions on a four-point Likert scale, with a total score ranging between 0 and 24 . Higher scores indicate higher degrees of inattention. The mean total score is calculated by dividing the total or partial raw score by the number of questions (i.e., eight), while previous studies reported a satisfactory level of internal consistency at Cronbach's $\alpha=0.84-0.95$.

\section{The DSM-5 Level 2 Cross-Cutting Symptom}

\section{Measure-anger for parents and guardians (PROMIS}

Emotional Distress-Calibrated Anger Measure)

PROMIS-SF was developed by the National Institutes of Health (NIH), and in this study, the Korean version of the PROMIS Emotional Distress, Anger, as translated by Shin et al. [19] was used. PROMIS is a rating scale for parents and guardians regarding the severity of emotions such as irritability, anger, or becoming enraged during 2 weeks. It includes five questions on a five-point Likert scale, with a total score ranging between 5 and 25 . Higher scores indicate higher severity of anger. The raw scores of the five questions are added to produce the total raw score, and the respective $\mathrm{T}$ score is checked. A T score $<55$ is considered normal; $\mathrm{T}$ score $\geq 55$ and $<60$ indicates a moderate level of anger; $\mathrm{T}$ score $\geq 60$ and $<70$ indicates a moderate level of anger; and a $\mathrm{T}$ score $\geq 70$ indicates a severe level of anger. The Cronbach's $\alpha$ at the time of development was 0.92 , and the Cronbach's $\alpha$ of the K-PROMIS Anger scale as translated by Choi et al. [20] is 0.91 .

\section{K-IOWA}

A ten-question scale developed by Loney and Milich [23],
K-IOWA, is used for the screening of ADHD and the evaluation of treatment effects [24]. In this study, the K-IOWA translated by Shin et al. [15] was used, and its reliability and validity have previously been investigated. The K-IOWA consists of two subcategories: inattentive/overactive and oppositional/defiant, each containing five questions. It includes ten questions on a four-point Likert scale, with a total score ranging between 0 and 30 , and higher scores indicate higher severity of inattentive-oppositional defiant behaviors. The Cronbach's $\alpha$ of K-IOWA is 0.91 .

\section{K-ARS}

ARS was developed by DuPaul [13] as a scale to assess children's behaviors, and the scale was designed to assess ADHD symptoms in school-age children based on the ADHD diagnostic criteria of DSM-IV. In this study, the Korean version (K-ARS), as translated by Kim et al. [14], was used, and its reliability and validity have previously been investigated. The K-ARS comprises 18 questions in total; the total score of odd questions measures inattentiveness, and the total score of even questions measures the hyperactivity/impulsivity. Each question is rated on a three-point Likert scale according to the frequency of the respective problem behavior. The reliability and validity of the scale have been verified in previous studies. In the study by Kim et al. [14], a satisfactory internal consistency at Cronbach's $\alpha=0.83-0.96$, with the range being dependent on age, was found.

\section{Statistical analysis}

For all statistical analyses, SPSS 20.0 (IBM Corp., Armonk, NY, USA) was used. The reliability of the Korean versions of the DSM-5 SNAP-IV inattention scale and PROMIS Anger scale was assessed by calculating the internal consistency coefficient. To examine the concurrent validity of the SNAP-IV inattention scale, the correlation coefficients with the K-IOWA inattention scale and K-ARS inattention scale were calculated. For the concurrent validity of the PROMIS Anger scale, the correlation coefficient with K-IOWA opposition$\mathrm{al} / \mathrm{defiant}$ scale was calculated. In addition, the construct validity of the SNAP-IV inattention scale and PROMIS Anger scale was examined through factor analysis to verify whether each scale could validly assess the constructs of inattention and anger.

\section{RESULTS}

\section{Reliability}

The internal consistency coefficient (Cronbach's $\alpha$ ) of the Korean version of the SNAP-IV inattention scale was 0.94, and that of the Korean version of the PROMIS Anger scale 
was 0.91 , indicating considerably high levels of consistency. The questions of the two scales are highly homogeneous, and the scales can thus be presumed to ensure a reliable assessment of the symptoms of anger and inattention (Table 1).

\section{Concurrent validity}

\section{Concurrent validity for the K-SNAP-IV, K-IOWA, and $\mathrm{K}$-ARS inattention scales}

To examine the concurrent validity of the Korean version of the SNAP-IV inattention scale, the correlations with the $\mathrm{K}$-IOWA inattention and K-ARS inattention scales were analyzed, and the correlation coefficients were found to be 0.84 and 0.86 , respectively, indicating significant correlations. The results proved that K-SNAP-IV could assess inattention in children and adolescents as reliably as the conventional KIOWA and K-ARS inattention scales with verified reliability and validity (Table 2).

\section{Concurrent validity for the PROMIS Anger scale, \\ $\mathrm{K}$ - IOWA, and K-ARS}

To examine the concurrent validity of the Korean version of the PROMIS Anger scale, correlations with the K-IOWA

Table 1. Internal consistency coefficient of SANP-IV inattention, and PROMIS Anger scales

\begin{tabular}{lc}
\hline SANP-IV inattention & Cronbach's $\alpha$ \\
PROMIS Anger & 0.94 \\
\hline SNAP-IV: & 0.91
\end{tabular}

SNAP-IV: the DSM-5 Level 2 Cross-Cutting Symptom Measure-inattention for parents and guardians (Swanson, Nolan and Pelham, version IV), PROMIS: Patient-Reported Outcome Measurement Information System

Table 2. Correlation coefficients of SNAP-IV, IOWA, and K-ARS inattention scales $(n=104)$

\begin{tabular}{lccc}
\hline & $\begin{array}{c}\text { SNAP-IV } \\
\text { inattention }\end{array}$ & $\begin{array}{c}\text { IOWA } \\
\text { inattention }\end{array}$ & $\begin{array}{c}\text { K-ARS } \\
\text { inattention }\end{array}$ \\
\hline SNAP-IV inattention & 1.00 & & \\
IOWA inattention & $0.84^{*}$ & 1.00 & \\
K-ARS inattention & $0.86^{*}$ & $0.73^{*}$ & 1.00 \\
\hline
\end{tabular}

${ }^{*} \mathrm{p}<0.01$. SSNAP-IV: the DSM-5 Level 2 Cross-Cutting Symptom Measure-inattention for parents and guardians (Swanson, Nolan and Pelham, version IV), IOWA: IOWA Conners Rating Scale, KARS: the Korean version of Attention-Deficit/Hyperactivity Disorder Rating Scales
Oppositional/Defiant scale and K-ARS hyperactive/impulsive scale were analyzed, and the correlation coefficients were found to be 0.75 and 0.47 , respectively. This indicated that the K-PROMIS could assess the oppositional and defiant behaviors of the children and adolescents as reliably as the conventional K-IOWA oppositional/defiant scale. The result also implied that the anger assessed by K-PROMIS slightly differed from the hyperactive/impulsive behaviors assessed by K-ARS (Table 3). Therefore, the PROMIS Anger scale cannot validly evaluate hyperactivity/impulsivity, which are core symptoms of ADHD, but it seems to be a good test for evaluating anger control problems in children with ADHD and for re-evaluating problems after treatment.

\section{Discriminant validity}

To examine the discriminant validity of the SNAP-IV inattention and PROMIS Anger scales, the mean scores of the two scales of the five clinical groups categorized based on the DSM-V diagnostic criteria, namely 1) depressive disorder 2) anxiety, 3) ADHD, 4) ADHD+anxious depression, and 5) tic disorder+somatic symptoms, were compared (Table 4). The one-way analysis of variance (ANOVA) performed on mean scores of the SNAP-IV inattention scale for the clinical groups showed that the mean differences per group were significant $(\mathrm{F}=8.58, \mathrm{p}<0.001)$. The Bonferroni test as a post-hoc test showed that the mean scores of the ADHD+anxious depression group were significantly higher than the mean scores of the tic disorder+somatic symptoms group, while the mean scores on the inattention scale were significantly higher for the ADHD group than for the anxiety and tic disorder+somatic symptoms groups $(4>5 ; 3>2,5)$. On the other hand, there was no significant difference in the mean scores of the SNAP-IV inattention scale between the ADHD and the depressive disorder groups, suggesting that children with depression show inattentive symptoms that are similar to those shown by children with ADHD. However, this may be the result of the fact that the sample size of the depressive disorder group is smaller than that of the pure ADHD or ADHD with anxious depression. Therefore, there is a need to conduct a clinical study with a larger sample size to verify the result in the future. One-way ANOVA on the PROMIS Anger scale for clinical groups showed that the mean score of the ADHD+ anxious depression group tended to be higher than that of

Table 3. Correlation of PROMIS Anger, IOWA oppositional/defiant, and K-ARS hyperactive/impulsive scales (n=104)

\begin{tabular}{lcc}
\hline & PROMIS Anger & IOWA oppositional/defiant \\
\hline PROMIS Anger & 1.00 & K-ARS hyperactive/impulsive \\
IOWA oppositional/defiant & $0.75^{*}$ & 1.00 \\
K-ARS hyperactive/impulsive & $0.47^{*}$ & $0.63^{*}$ \\
\hline
\end{tabular}

${ }^{*} \mathrm{p}<0.01$. PROMIS: Patient-Reported Outcome Measurement Information System, IOWA: IOWA Conners Rating Scale, K-ARS: the Korean version of Attention-Deficit/Hyperactivity Disorder Rating Scales 
Table 4. Comparison of mean score in SNAP-IV inattention and PROMIS Anger scales by groups $(n=104)$

\begin{tabular}{|c|c|c|c|c|c|c|c|}
\hline & $\begin{array}{c}\text { Depression }^{a} \\
(n=9)\end{array}$ & $\begin{array}{c}\text { Anxiety disorder } \\
(n=14)\end{array}$ & $\begin{array}{l}\text { ADHD }^{c} \\
(n=41) \\
\end{array}$ & $\begin{array}{c}\text { ADHD } \text { anxious } \\
\text { depression }(n=11)\end{array}$ & $\begin{array}{c}\text { Tic+somatic }{ }^{e} \\
\text { symptom }(n=29)\end{array}$ & $\mathrm{F}$ & $\begin{array}{l}\text { Post } \\
\text { hoc }\end{array}$ \\
\hline \multirow[t]{2}{*}{ SNAP-IV inattention } & $6.89(5.90)$ & $6.86(6.11)$ & $11.90(5.40)$ & $12.64(7.27)$ & $5.03(4.53)$ & $8.58^{*}$ & $d>e$ \\
\hline & & & & & & & $c>b, e$ \\
\hline PROMIS Anger & $4.56(2.55)$ & $6.64(4.27)$ & $6.68(5.51)$ & $7.18(4.40)$ & $3.69(3.67)$ & 2.45 & \\
\hline
\end{tabular}

Data are presented as mean (standard deviation). ${ }^{*} \mathrm{p}<0.001$. SNAP-IV: the DSM-5 Level 2 Cross-Cutting Symptom Measure-inattention for parents and guardians (Swanson, Nolan and Pelham, version IV), PROMIS: Patient-Reported Outcome Measurement Information System, ADHD: attention-deficit/hyperactivity disorder

Table 5. Factor loadings of the DSM-5 Level 2 Cross-Cutting Symptom Measure-inattention for parents and guardians (Swanson, Nolan and Pelham, version IV) rating scale ( $\mathrm{n}=104)$

\begin{tabular}{lr}
\hline \multicolumn{1}{c}{ Questions } & Factor loading \\
\hline $\begin{array}{l}\text { 1. My child often fails to give close attention to details or makes careless mistakes in schoolwork, work, } \\
\text { or other activities }\end{array}$ & 0.874 \\
2. My child often has difficulty sustaining attention in tasks or play activities & 0.868 \\
3. My child often does not seem to listen when spoken to directly & 0.748 \\
4. My child often does not follow through on instructions and fails to finish schoolwork, chores, or duties & 0.880 \\
5. My child often has difficulty organizing tasks and activities & 0.877 \\
6. My child often avoids, dislikes, or is reluctant to engage in tasks that require sustained mental effort & 0.839 \\
(e.g., schoolwork or homework) & 0.739 \\
7. My child often loses things necessary for tasks or activities (e.g., toys, school assignments, pencils, books, \\
or tools)
\end{tabular}

the other four groups. However, the mean differences among the groups were not significant. These results showed that the discriminant validity of the PROMIS Anger scale is low, making it difficult to discriminate children with ADHD from children with other psychiatric disorders such as depression, anxiety, and tic disorder using the PROMIS Anger scale.

\section{Construct validity}

Factor analysis was performed on all data collected through SNAP-IV inattention and PROMIS Anger scales from 104 children and adolescents in this study. When principal component analysis was used to extract the factors, both scales were shown to be unidimensional with a single factor, 'inattention' or 'anger.' The construct validity of the SNAP-IV inattention and PROMIS Anger scales was thus verified (Tables 5 and 6). Among the eight questions of the SNAP-IV inattention scale, the following were shown to most effectively assess inattention: "my child often does not follow through on instructions and fails to finish schoolwork, chore, or duties," "my child often has difficulty organizing tasks and activities," and "my child often fails to give close attention to details or makes careless mistakes in schoolwork, work, and other activities." Among the five questions of the PROMIS Anger scale, "my child was so angry that he/she felt like yelling at somebody" and "my child felt mad" were shown to most effectively assess anger.
Table 6. Factor loadings of Patient-Reported Outcome Measurement Information System Anger scale $(n=104)$

\begin{tabular}{lc}
\hline \multicolumn{1}{c}{ Questions } & Factor loading \\
\hline 1. My child felt mad & 0.884 \\
2. My child was so angry he/she felt like & 0.930 \\
$\quad$ yelling at somebody & \\
3. My child was so angry he/she felt like & 0.877 \\
$\quad$ throwing something & \\
4. My child felt upset & 0.792 \\
5. When my child got mad, he/she stayed & 0.833 \\
$\quad$ mad & \\
Eigenvalues & 3.73 \\
\hline
\end{tabular}

\section{DISCUSSION}

This study investigated the reliability and validity of the Korean version of the SNAP-IV inattention scale and the PROMIS Anger scale for parents and guardians of children and adolescents aged 6-17 years. The reliability and validity of the information on the behaviors of children and adolescents provided by parents and guardians through the SNAPIV inattention scale and the PROMIS Anger scale have been shown to be high in previous studies in other countries [23, $25,26]$, while the scales were also shown to be useful in assessing the treatment effects or progress. Notably, they are time-efficient assessments of the problematic behaviors of 
children and adolescents, as ratings are performed in a short period. The two scales are thus expected to be useful in clinical settings where repeated measurements are required to verify the treatment effects.

The reliability coefficient of the Korean version of the SNAP-IV inattention scale was shown to be remarkably high at Cronbach's $\alpha=0.94$. The SNAP-IV inattention scale can thus be regarded as a homogeneous scale that ensures a highly reliable assessment of inattention, in particular, among the ADHD-related symptoms. Moreover, the correlation coefficients for the SNAP-IV and the inattentive/overactive score of the K-IOWA and inattention score of the K-ARS were 0.84 and 0.86 , respectively, indicating high levels to support the concurrent validity of the SNAP-IV inattention scale. The children and adolescents in the ADHD+anxious depression group and the ADHD group showed significantly higher scores than the other groups on the SNAP-IV inattention scale, implying that the scale can differentiate children and adolescents with ADHD-related problems from those with other psychiatric disorders such as depression, anxiety, or tic disorder. Thus, the findings suggested that the SNAP-IV inattention scale can be not only an easy tool to use but also a reliable and valid test in treatment evaluation as well as differential screening for children and adolescents with ADHD.

The reliability coefficient of the Korean version of the PROMIS Anger scale was shown to be remarkably high at Cronbach's $\alpha=0.91$. The PROMIS Anger scale can thus be regarded as a homogeneous scale for reliably assessing anger. Moreover, the correlation coefficients for the PROMIS and the impulsive/hyperactive score of the K-ARS and the oppositional/defiant score of the K-IOWA were 0.47 and 0.75 , respectively. These results suggest that the construct assessed by the PROMIS Anger scale was related to the oppositional and defiant characteristics [27] that are regarded as an attitude and behavioral response to the stimulus causing anger. The construct of the PROMIS was different from impulsive/ hyperactive behaviors as the core symptoms of ADHD. In addition, the result of the analysis of the group differences in mean scores of the PROMIS Anger scale showed a trend of higher anger scores in the groups of pure ADHD or ADHD with anxiety/depression than in the depression or tic disorder+ somatic symptoms groups. Children and adolescents with ADHD repeatedly experience feelings of frustration in daily life, including in academics and social relationships [4], which may cause emotional problems such as anxiety or depression; the result may thus be taken to imply that such problems could lead to secondary behavioral problems involving oppositional and defiant behaviors. Furthermore, the mean anger score of the anxiety group was almost as high as that of the ADHD group. This may imply that anxiety in child- hood and adolescence may cause emotional problems related to suppressed anger [28]. However, as the sample sizes of the anxiety and depression groups were smaller than those of the groups of pure ADHD or ADHD with anxiety/depression, the results should be verified in further studies recruiting a larger number of clinical groups. In previous studies $[29,30]$, a high comorbidity of ADHD and tic disorder was reported, but the lowest inattention and anger scores were shown by the tic+somatic symptoms group in this study because we did not recruit patients with ADHD and tic disorder. In future studies, it is necessary to recruit children with ADHD with tic disorder to compare inattention and anger problems in children with ADHD and those in children with ADHD with tic disorder.

The factor analysis shows that the SNAP-IV inattention scale is a unidimensional scale of a set of questions for a single factor (inattention), suggesting that the scale might be useful for assessing children and adolescents with inattentive type of ADHD.

Nonetheless, a number of things should be considered when using the SNAP inattention scale or the PROMIS Anger scale. First, the two scales for parents and guardians cannot replace the direct diagnostic tests on children and adolescents, even when high scores have been obtained in these scales. A more accurate assessment and diagnosis is required based on a higher level of expertise and clinical judgment by a clinician. Furthermore, as most rating scales evaluate quantitative differences such as severity rather than the qualitative difference of symptomatic expression, the various unique characteristics of each child's symptoms should be taken into consideration.

Finally, the limitations of this study are as follows. This study investigated the reliability and validity of the Korean version of the SNAP-IV inattention scale and the PROMIS Anger scale by comparing different clinical groups visiting the department of child and adolescent psychiatry. However, the number of participants was relatively small. In addition, the pattern of inattention and anger may vary according to the gender and age of children and adolescents. Further studies should be conducted to establish the norm scores on each scale based on age and gender for a more objective analysis of severity. Lastly, the scales examined in this study are rating scales for parents and guardians; therefore, there is a possibility that the subjective discomfort and severity of experience in children and adolescents with ADHD could not be sufficiently reflected. Thus, the DSM-5 Level 2 self-reporting scale for children and adolescents and the rating scale for parents and guardians should be applied together to further increase their utility as tools for screening and treatment. 


\section{CONCLUSION}

The reliability and validity of the Korean version of DSM5 Level 2 Cross-cutting Symptom Measure inattention and anger for parents and guardians of children aged 6-17 years were examined by targeting the parents and guardians of the clinical group of children and adolescents visiting the department of child and adolescent psychiatry at a university hospital located in Seoul, Korea. The results showed high levels of reliability and validity for the two scales, which are expected to be useful, in particular, as tools for not only an efficient screening of ADHD in clinical practice but also for better differentiation of children and adolescents with ADHD from those with emotional problems such as depression, anxiety, or tic disorder. The findings suggested that the DSM-5 inattention and anger scales for parents and guardians would be useful in clinical settings, mental health care service, and research as easy, economic assessment tools.

\section{Acknowledgments}

This study was conducted by the fund of the Korean Ministry of Health and Welfare (800-20200053).

\section{Conflicts of Interest}

The authors have no potential conflicts of interest to disclose.

\section{Author Contributions}

Conceptualization: Min-Sup Shin. Data curation: all authors. Formal analysis: all authors. Funding acquisition: Min-Sup Shin, Bung-Nyun Kim. Investigation: all authors. Methodology: Min-Sup Shin, Minji Cho, Mirae Jang, Hanbyul Shin. Project administration: Min-Sup Shin, Minji Cho, Mirae Jang, Hanbyul Shin. Resources: all authors. Supervision: Min-Sup Shin. Writing_original draft: Min-Sup Shin, Minji Cho, Mirae Jang, Hanbyul Shin. Writing_review \& editing: Min-Sup Shin, Minji Cho, Mirae Jang, Hanbyul Shin.

\section{ORCID iDs}

Min-Sup Shin

Bung-Nyun Kim

Minji Cho

Mirae Jang

Hanbyul Shin

Ryemi Do

Hyungseo Park

Narae Yoon

Gahye Noh

Jae-Won Song

Yebin Ahn

Jiyoon Shin

Soomin Jang

Eunjung Noh

Eunhwa Lee https://orcid.org/0000-0001-9840-6997 https://orcid.org/0000-0002-2403-3291 https://orcid.org/0000-0003-2837-6774 https://orcid.org/0000-0002-6106-8370 https://orcid.org/0000-0002-3919-3466 https://orcid.org/0000-0003-3613-9955 https://orcid.org/0000-0002-1804-5595 https://orcid.org/0000-0002-5415-4617 https://orcid.org/0000-0002-4225-4266 https://orcid.org/0000-0002-3109-3794 https://orcid.org/0000-0001-9566-5555 https://orcid.org/0000-0003-0510-993X https://orcid.org/0000-0003-3515-7211 https://orcid.org/0000-0002-0834-5543 https://orcid.org/0000-0002-6142-1365

\section{REFERENCES}

1) Park S, Kim BN, Cho SC, Kim JW, Shin MS, Yoo HJ. Prevalence, correlates, and comorbidities of DSM-IV psychiatric disorders in children in Seoul, Korea. Asia Pac J Public Health 2015;27:NP1942NP1951.

2) Health Insurance Review \& Assessment Service. Trend of the number of patients per year for ADHD in Korea [cited 2020 Oct 20]. Available from URL: http://opendata.hira.or.kr/op/opc/olapMfrnIntrsIlnsInfo.do.

3) American Psychiatric Association. Diagnostic and Statistical Manual of Mental disorders (DSM- $5^{\circledR}$ ). 5th ed. Arlington, VA: American Psychiatric Association;2013.

4) Raggi VL, Chronis AM. Interventions to address the academic impairment of children and adolescents with ADHD. Clin Child Fam Psychol Rev 2006;9:85-111.

5) Edwards G, Barkley RA, Laneri M, Fletcher K, Metevia L. Parentadolescent conflict in teenagers with ADHD and ODD. J Abnorm Child Psychol 2001;29:557-572.

6) Baren M. ADHD in adolescents: will you know it when you see it? Contemp Pediatr 2002;19:124-143.

7) Kitchens SA, Rosén LA, Braaten EB. Differences in anger, aggression, depression, and anxiety between ADHD and non-ADHD children. J Atten Disord 1999;3:77-83.

8) Harty SC, Miller CJ, Newcorn JH, Halperin JM. Adolescents with childhood ADHD and comorbid disruptive behavior disorders: aggression, anger, and hostility. Child Psychiatry Hum Dev 2009;40: 85-97.

9) Abikoff HB, Jensen PS, Arnold LLE, Hoza B, Hechtman L, Pollack S, et al. Observed classroom behavior of children with ADHD: relationship to gender and comorbidity. J Abnorm Child Psychol 2002; 30:349-359.

10) Danckaerts M, Heptinstall E, Chadwick O, Taylor E. Self-report of attention deficit and hyperactivity disorder in adolescents. Psychopathology 1999;32:81-92.

11) Teicher MH, Ito Y, Glod CA, Barber NI. Objective measurement of hyperactivity and attentional problems in ADHD. J Am Acad Child Adolesc Psychiatry 1996;35:334-342.

12) Janicke DM, Finney JW, Riley AW. Children's health care use: a prospective investigation of factors related to care-seeking. Med Care 2001;39:990-1001.

13) DuPaul GJ. Parent and Teacher Rating of ADHD symptoms: Psychometric Properties in a Community-Based Sample. J Clin Child Adolesc Psychol 1991;20:245-253.

14) Kim YS, So YK, Noh JS, Choi NK, Kim SJ, Koh YJ. Normative data on the Korean ADHD Rating Scales (K-ARS) for parents and teacher. J Korean Neuropsychiatr Assoc 2003;42:352-359.

15) Shin MS, Ryu ME, Kim BN, Hwang JW, Cho SC. Development of the Korean version of the IOWA Conners Rating Scale. J Korean Neuropsychiatr Assoc 2005;44:82-88.

16) Oh KJ, Lee HL. Assessment of ADHD with abbreviated Conners Rating Scale. Korean J Clin Psychol 1989;8:135-142.

17) American Psychiatric Association. Diagnostic and statistical manual of mental disorders: DSM-5. 5th ed. Arlington, VA: American Psychaitric Association;2013.

18) Narrow WE, Clarke DE, Kuramoto SJ, Kraemer HC, Kupfer DJ, Greiner L, et al. DSM-5 field trials in the United States and Canada, part III: development and reliability testing of a cross-cutting symptom assessment for DSM-5. Am J Psychiatry 2013;170:71-82.

19) American Psychiatric Association. Handbook of DSM-5 diagnostic assessment measures. Shin MS, Oh SJ, Kwon JS, trans. Seoul: Hakjisa;2019.

20) Choi H, Kim C, Ko H, Park CG. Translation and validation of the Korean version of PROMIS $®$ pediatric and parent proxy measures for emotional distress. J Patient Rep Outcomes 2019;3:36.

21) Kim Y, Kim B, Chang JS, Kim BN, Cho SC, Hwang JW. Parental 
quality of life and depressive mood following methylphenidate treatment of children with attention-deficit hyperactivity disorder. Psychiatry Clin Neurosci 2014;68:506-514.

22) Swanson JM. School-Based Assessment and Interventions for ADD students. Irvine, CA: KC Publishing; 1992.

23) Hall CL, Guo B, Valentine AZ, Groom MJ, Daley D, Sayal K, et al. The validity of the SNAP-IV in children displaying ADHD symptoms. Assessment 2020;27:1258-1271.

24) Loney K, Milich R. Hyperactivity, inattention, and aggression in clinical practice. In: Wolraich M, Routh DK, editors. Adcances in behavioral pediatrics. Greenwich, CT: JAI Press;1982.

25) Costa DS, de Paula JJ, Malloy-Diniz LF, Romano-Silva MA, Miranda DM. Parent SNAP-IV rating of attention-deficit/hyperactivity disorder: accuracy in a clinical sample of ADHD, validity, and reliability in a Brazilian sample. J Pediatr (Rio J) 2019;95:736-743.

26) Junghaenel DU, Schneider S, Stone AA, Christodoulou C, Broder- ick JE. Ecological validity and clinical utility of Patient-Reported Outcomes Measurement Information System (PROMIS ${ }^{\circledR}$ ) instruments for detecting premenstrual symptoms of depression, anger, and fatigue. J Psychosom Res 2014;76:300-306.

27) Seo SG, Kwon SM. Relations of self-esteem and narcissism with aggressiveness. Kor J Clin Psychol 2002;21:809-826.

28) Walsh LM, Wolk CB, Haimes EMB, Jensen-Doss A, Beidas RS. The relationship between anger and anxiety symptoms in youth with anxiety disorders. J Child Adolesc Couns 2018;4:117-133.

29) Robertson MM. Attention deficit hyperactivity disorder, tics and Tourette's syndrome: the relationship and treatment implications. A commentary. Eur Child Adolesc Psychiatry 2006;15:1-11.

30) Biederman J, Kwon A, Aleardi M, Chouinard VA, Marino T, Cole $\mathrm{H}$, et al. Absence of gender effects on attention deficit hyperactivity disorder: findings in nonreferred subjects. Am J Psychiatry 2005; 162:1083-1089. 